\title{
On the error term of an asymptotic formula of Ramanujan
}

\author{
by \\ H. Maier (Ulm) and A. Sankaranarayanan (Mumbai) \\ Dedicated to Professor Yoichi Motohashi \\ on his sixtieth birthday
}

1. Introduction. In [21], Ramanujan records (without proof) many curious asymptotic formulae. One of them is

$$
\begin{aligned}
d^{2}(1)+d^{2}(2)+\cdots+d^{2}(n)= & A n(\log n)^{3}+B n(\log n)^{2}+C n \log n \\
& +D n+O\left(n^{3 / 5+\varepsilon}\right) .
\end{aligned}
$$

Also he records (without proof) the result that on the assumption of the Riemann hypothesis, the error term in (1.1) can be improved to $O\left(n^{1 / 2+\varepsilon}\right)$. In view of a method due to H. L. Montgomery and R. C. Vaughan (see [17]), it is very likely that the error term is $O\left(n^{1 / 2}\right)$. We propose this as a conjecture (see also [19], [22]). Unconditionally, the error term related to $d^{2}(j)$ is known to be $O\left(n^{1 / 2+\varepsilon}\right)$ for any positive constant $\varepsilon$ (see for example equation (14.30) of [10] and also [5] and [28]). Professor A. Schinzel has already considered some of the problems of Ramanujan (see [24]), namely for the arithmetic function $r^{2}(n)$, and he has proved that the corresponding error term is $\Omega\left(n^{3 / 8}\right)$. Also the corresponding error term is $O\left(n^{1 / 2}(\log n)^{8 / 3}(\log \log n)^{1 / 3}\right)$ which is due to M. Kühleitner and W. G. Nowak (see [15], [16]). Let

$$
E(x)=\sum_{n \leq x} d^{2}(n)-x P_{3}(\log x),
$$

where $P_{3}(y)$ is a polynomial in $y$ of degree 3 . From a general theorem of M. Kühleitner and W. G. Nowak (see e.g. (5.4) of [15]), it follows that

$$
E(x)=\Omega\left(x^{3 / 8}\right) .
$$

2000 Mathematics Subject Classification: Primary 11M; Secondary 11M06, 11N05.

Key words and phrases: error term, Riemann zeta-function, Möbius function, Minkowski's inequality. 
Let

$$
\sum_{n \leq x} d_{4}(n)=a x(\log x)^{3}+b x(\log x)^{2}+c x(\log x)+d x+O\left(x^{\alpha}\right) .
$$

Assuming that $\alpha<1 / 2$ in (1.3), D. Suryanarayana and R. Sitaramachandra rao (see [25]) showed that (with some $A>0$ )

$$
E(x) \ll x^{1 / 2} \exp \left(-A(\log x)^{3 / 5}(\log \log x)^{-1 / 5}\right),
$$

and assuming additionally the Riemann hypothesis, they established (see [25]) that

$$
E(x) \ll x^{\frac{2-\alpha}{5-4 \alpha}} \exp \left(A(\log x)(\log \log x)^{-1}\right) .
$$

In [20], the second author jointly with K. Ramachandra proved that unconditionally, we have

$$
E(x) \ll x^{1 / 2}(\log x)^{5}(\log \log x) .
$$

It should be mentioned that recently M. Kühleitner and W. G. Nowak (see [16]) have given a precise upper bound for the error term related to the average number of solutions of the Diophantine equation $u^{2}+v^{2}=w^{3}$, and their arguments are in fact more general. For some more general interesting results, we refer to for example [1], [2], [3] and [23]; we also mention some related references [4], [14] and [27].

The main aim of this paper is to prove:

For $Y \geq Y_{0}$, we have (unconditionally)

$$
\frac{1}{Y} \int_{Y}^{2 Y}(E(x))^{2} d x \ll Y \exp \left(-C(\log Y)^{3 / 5}(\log \log Y)^{-1 / 5}\right)
$$

for an effective positive constant $C$.

That is, the natural but unproven conjectural inequality (1.4) is true in mean-square. This is established in a more general frame involving the integers $k, l$ in Theorem 2 below.

Let $k \geq 2$ and $l \geq 2$ be integers. We define the Dirichlet series (in $\sigma>1$ )

$$
F(s)=\frac{\zeta^{k}(s)}{\zeta(l s)}=\sum_{n=1}^{\infty} b_{n} n^{-s} .
$$

Then, from the Perron formula (see for example [18]), we obtain

$$
\begin{aligned}
\sum_{n \leq x} b_{n}= & A_{(k-1)} x(\log x)^{k-1}+A_{(k-2)} x(\log x)^{k-2} \\
& +A_{(k-3)} x(\log x)^{k-3}+\cdots+A_{(0)} x+E_{k, l}(x) \\
=: & M_{k, l}(x)+E_{k, l}(x),
\end{aligned}
$$




$$
\begin{aligned}
\sum_{n \leq x} d_{k}(n)= & D_{(k-1)} x(\log x)^{k-1}+D_{(k-2)} x(\log x)^{k-2} \\
& +D_{(k-3)} x(\log x)^{k-3}+\cdots+D_{(0)} x+\Delta_{k}(x) \\
= & : M_{k}(x)+\Delta_{k}(x) .
\end{aligned}
$$

Note that the coefficients $A_{(j)}$ in (1.8) will depend on $l$ whereas $D_{(j)}$ in (1.9) are independent of $l$.

We study the more general error term $E_{k, l}(x)$ of the Ramanujan type. We define

$$
\alpha_{k}:=\inf \left\{\alpha: \Delta_{k}(x) \ll x^{\alpha}\right\}
$$

and

$$
\beta_{k}:=\inf \left\{\beta: \int_{2}^{Y}\left(\Delta_{k}(x)\right)^{2} d x \ll Y^{1+2 \beta}\right\} .
$$

It is already known from the work of Kolesnik (see [13] and [12] respectively) that $\alpha_{2} \leq 139 / 429, \alpha_{3} \leq 43 / 96$ (a better value of $\alpha_{2} \leq 23 / 73$ is known from the work of M. N. Huxley (see [9]) and in fact $\alpha_{2} \leq 131 / 416$ from an unpublished work of M. N. Huxley), and from the work of D. R. HeathBrown (see [7] and [8]) that

$$
\alpha_{k}= \begin{cases}3 / 4-1 / k & \text { for } 4 \leq k \leq 8 \\ 1-3 / k & \text { for } k \geq 8\end{cases}
$$

Better upper bounds are available for certain intermediate values of $k$ (see Theorem 13.2 of [10]), namely $\alpha_{9} \leq 35 / 54, \alpha_{10} \leq 41 / 60, \alpha_{11} \leq 7 / 10$ and $\alpha_{12} \leq 5 / 7$.

General Conjecture. For every integer $k \geq 2$, we have

$$
\alpha_{k}=\frac{k-1}{2 k}
$$

Regarding $\beta_{k}$, first of all we observe that $\beta_{k} \leq \alpha_{k}$. It is already known that (see Theorem 12.6(A) of [26])

$$
\beta_{k} \geq \frac{k-1}{2 k} \text {. }
$$

We also know (see Theorem 12.8 of [26] and also Theorems 13.9 and 13.10 of [10]) that $\beta_{2}=1 / 4, \beta_{3}=1 / 3$, and from the work of D. R. Heath-Brown (see [7] and [8]) that $\beta_{4}=3 / 8$. We should also mention a result of Jutila (see [11]) which states that if $\alpha_{2}=1 / 4$, then $\mu(1 / 2) \leq 3 / 20$ and $E^{*}(T) \ll$ $T^{5 / 16+\varepsilon}$, where $\mu(1 / 2)=\inf \left\{\xi: \zeta(1 / 2+i t) \ll(|t|+10)^{\xi}\right\}$ and

$$
\int_{0}^{T}\left|\zeta\left(\frac{1}{2}+i t\right)\right|^{2} d t=T \log \left(\frac{T}{2 \pi}\right)+(2 \gamma-1) T+E^{*}(T) .
$$

Throughout the paper, we write

$$
\delta(x):=\exp \left(-A(\log x)^{3 / 5}(\log \log x)^{-1 / 5}\right)
$$


with $A$ being a positive constant, and we assume that $x \geq e^{l e^{l}}$ and $Y \geq$ $100 e^{l e^{l}}$. We prove

Theorem 1. For every $\varepsilon>0$ and for $x \geq x_{0}(l)$, we have

$$
E_{k, l}(x) \ll \begin{cases}x^{\alpha_{k}+2 \varepsilon} & \text { if } l \alpha_{k} \geq 1-l \varepsilon \\ x^{1 / l} & \text { if } l \alpha_{k}<1-l \varepsilon\end{cases}
$$

Theorem 2. For every $\varepsilon>0$ and $Y \geq Y_{0}(l)$, we have

$$
\begin{aligned}
I & =\frac{1}{Y} \int_{Y}^{2 Y}\left(E_{k, l}(x)\right)^{2} d x \\
& \ll \begin{cases}Y^{2 \beta_{k}+2 \varepsilon} & \text { if } l \beta_{k} \geq 1-l \varepsilon / 2, \\
Y^{2 / l} \exp \left(-C(\log Y)^{3 / 5}(\log \log Y)^{-1 / 5}\right) & \text { if } l \beta_{k}<1-l \varepsilon / 2,\end{cases}
\end{aligned}
$$

where $C$ is an effective positive constant depending only on $k, l$ and $\varepsilon$.

2. Notation and preliminaries. $C$ and $A$ (with or without suffixes) denote effective positive constants unless otherwise specified, which need not be the same at each occurrence. We write $f(x) \ll g(x)$ to mean $|f(x)|<$ $C_{1} g(x)$ (sometimes we use the $O$ notation also). The notation $[x]$ denotes the integral part of $x$. The implied constants are all effective. We assume that $x \geq x_{0}(l)$ and $Y \geq Y_{0}(l)$ where $x_{0}(l)$ and $Y_{0}(l)$ are positive constants depending only on $l$.

\section{Some lemmas}

Lemma 3.1. We have the relation

$$
b_{n}=\sum_{j^{l} \mid n} \mu(j) d_{k}\left(\frac{n}{j^{l}}\right) .
$$

Proof. The proof is obvious.

Lemma 3.2. For $s>1$ and $r \geq 0$, we have

$$
\sum_{n \leq x} n^{-s} \mu(n)(\log n)^{r}=(-1)^{r} \eta^{(r)}(s)+O\left(x^{-(s-1)} \delta(x)(\log x)^{r}\right),
$$

where $\eta^{(0)}(s)=\eta(s)=(\zeta(s))^{-1}$ and $\eta^{(r)}(s)$ for $r \geq 1$ denotes the rth derivative of $\eta(s)=(\zeta(s))^{-1}$.

Proof. This is Lemma 2.2 of [25].

Lemma 3.3. For $x \geq x_{0}(l)$, we have

$$
\ll\left|\sum_{n \leq \varrho x^{1 / l}} \mu(n) \Delta_{k}\left(\frac{x}{n^{l}}\right)\right|+x^{1 / l} \varrho^{1-l} \delta\left(\varrho x^{1 / l}\right)\left(\log \left(\max \left(\frac{1}{\varrho}, x\right)\right)\right)^{C_{k}},
$$


where $0<\varrho(=\varrho(x))<1$ and $C_{k}$ is an effective positive constant depending only on $k$.

Proof. We fix $z=x^{1 / l}$ and let $\varrho(=\varrho(x)$ ) be a number (or a function of $x$ ) which satisfies $0<\varrho<1$. We will choose $\varrho$ appropriately later. We notice that if $n^{l} r \leq x$, then both $n>\varrho z$ and $r>\varrho^{-l}$ cannot hold simultaneously, and hence

$$
\begin{aligned}
\sum_{n \leq x} b_{n} & =\sum_{\substack{n^{l} r \leq x \\
n \leq \varrho z}} \mu(n) d_{k}(r)+\sum_{\substack{n^{l} r \leq x \\
r \leq \varrho^{-l}}} \mu(n) d_{k}(r)-\sum_{\substack{n \leq \varrho z \\
r \leq \varrho^{-l}}} \mu(n) d_{k}(r) \\
& =: S_{1}+S_{2}-S_{3} .
\end{aligned}
$$

From (1.9), we have

$$
\begin{aligned}
& S_{1}=\sum_{\substack{n^{l} r \leq x \\
n \leq \varrho z}} \mu(n) d_{k}(r)=\sum_{n \leq \varrho z} \mu(n) \sum_{r \leq x n^{-l}} d_{k}(r) \\
= & \sum_{n \leq \varrho z} \mu(n)\left\{M_{k}\left(x n^{-l}\right)+\Delta_{k}\left(x n^{-l}\right)\right\} \\
= & \left\{D_{(k-1)} x(\log x)^{k-1}+D_{(k-2)} x(\log x)^{k-2}+\cdots+D_{(0)} x\right\} \\
& \times\left(\sum_{n \leq \varrho z} \mu(n) n^{-l}\right) \\
& -l x\left(D_{(k-1)}\left(\begin{array}{c}
k-1 \\
1
\end{array}\right)(\log x)^{k-2}+D_{(k-2)}\left(\begin{array}{c}
k-2 \\
1
\end{array}\right)(\log x)^{k-3}+\cdots\right) \\
& \times\left(\sum_{n \leq \varrho z} \mu(n) n^{-l}(\log n)\right) \\
& +l^{2} x\left(D_{(k-1)}\left(\begin{array}{c}
k-1 \\
2
\end{array}\right)(\log x)^{k-3}+D_{(k-2)}\left(\begin{array}{c}
k-2 \\
2
\end{array}\right)(\log x)^{k-4}+\cdots\right) \\
& \times\left(\sum_{n \leq \varrho z} \mu(n) n^{-l}(\log n)^{2}\right) \\
& -\cdots+(-1)^{k-1} D_{(k-1)} l^{k-1} x\left(\sum_{n \leq \varrho z} \mu(n) n^{-l}(\log n)^{k-1}\right) \\
& +\sum_{n \leq \varrho z} \mu(n) \Delta_{k}\left(x n^{-l}\right) .
\end{aligned}
$$

Applying Lemma 3.2 for $r=0,1, \ldots, k-1$ and $s=l$, we obtain

$$
\begin{aligned}
S_{1}= & \left\{D_{(k-1)} x(\log x)^{k-1}+D_{(k-2)} x(\log x)^{k-2}+\cdots+D_{(0)} x\right\} \\
& \times\left((\zeta(l))^{-1}+O\left((\varrho z)^{1-l} \delta(\varrho z)\right)\right)
\end{aligned}
$$




$$
\begin{aligned}
& -l x\left(D_{(k-1)}\left(\begin{array}{c}
k-1 \\
1
\end{array}\right)(\log x)^{k-2}+D_{(k-2)}\left(\begin{array}{c}
k-2 \\
1
\end{array}\right)(\log x)^{k-3}+\cdots\right) \\
& \times\left(-\eta^{(1)}(l)+O\left((\varrho z)^{1-l} \delta(\varrho z) \log (\varrho z)\right)\right) \\
& +l^{2} x\left(D_{(k-1)}\left(\begin{array}{c}
k-1 \\
2
\end{array}\right)(\log x)^{k-3}+D_{(k-2)}\left(\begin{array}{c}
k-2 \\
2
\end{array}\right)(\log x)^{k-4}+\cdots\right) \\
& \times\left(\eta^{(2)}(l)+O\left((\varrho z)^{1-l} \delta(\varrho z)(\log (\varrho z))^{2}\right)\right) \\
& -\cdots+(-1)^{k-1} D_{(k-1)} l^{k-1} x\left((-1)^{k-1} \eta^{(k-1)}(l)\right. \\
& \left.+O\left((\varrho z)^{1-l} \delta(\varrho z)(\log (\varrho z))^{k-1}\right)\right)+\sum_{n \leq \varrho z} \mu(n) \Delta_{k}\left(x n^{-l}\right) \\
& =M_{k, l}(x)+O\left(x(\varrho z)^{1-l} \delta(\varrho z)(\log x)^{k-1}\right)+\sum_{n \leq \varrho z} \mu(n) \Delta_{k}\left(x n^{-l}\right) .
\end{aligned}
$$

We find that

$$
\begin{aligned}
S_{2} & =\sum_{\substack{n^{l} r \leq x \\
r \leq \varrho^{-l}}} \mu(n) d_{k}(r)=\sum_{r \leq \varrho^{-l}} d_{k}(r) \sum_{n \leq(x / r)^{1 / l}} \mu(n) \\
& =\sum_{r \leq \varrho^{-l}} d_{k}(r) M\left((x / r)^{1 / l}\right) \\
& \ll x^{1 / l} \sum_{r \leq \varrho^{-l}} d_{k}(r) r^{-1 / l}\left(\delta\left(\left(\frac{x}{r}\right)^{1 / l}\right)\right) \\
& \ll x^{1 / l} \varrho^{1-l} \delta(\varrho z)\left(\log \left(\varrho^{-l}\right)\right)^{C_{k}},
\end{aligned}
$$

since $\left(\frac{x}{r}\right)^{1 / l}>\varrho z, \delta$ is decreasing, $\delta\left(\left(\frac{x}{r}\right)^{1 / l}\right) \leq \delta(\varrho z)$, and

$$
\sum_{r \leq \varrho^{-l}} d_{k}(r) r^{-1 / l}=\sum_{r \leq \varrho^{-l}} \frac{d_{k}(r)}{r} r^{1-1 / l} \ll \varrho^{1-l}\left(\log \left(\varrho^{-l}\right)\right)^{C_{k}} .
$$

We also notice that

$$
\begin{aligned}
S_{3} & =\sum_{\substack{n \leq \varrho z \\
r \leq \varrho^{-l}}} \mu(n) d_{k}(r)=\sum_{r \leq \varrho^{-l}} d_{k}(r) M(\varrho z) \\
& \ll \varrho^{-l}\left(\log \left(\varrho^{-l}\right)\right)^{C_{k}^{\prime}}(\varrho z) \delta(\varrho z) \\
& \ll x^{1 / l} \varrho^{1-l} \delta(\varrho z)\left(\log \left(\varrho^{-l}\right)\right)^{C_{k}^{\prime}}
\end{aligned}
$$

for $z=x^{1 / l}$. Now the lemma follows from (3.3.2) and (3.3.4)-(3.3.6). 


\section{Proof of the theorems}

Proof of Theorem 1. We choose $\varrho=1 / 10$ and note that $z=x^{1 / l}$. Therefore (from Lemma 3.3 and from the definition (1.10)), we obtain

$$
\begin{aligned}
& \ll_{k, l}(x) \\
& \ll_{l} \sum_{n \leq z / 10}\left|\Delta_{k}\left(\frac{x}{n^{l}}\right)\right|+x^{1 / l} \delta\left(\frac{z}{10}\right)(\log x)^{C_{k}} \\
& \ll_{l} \sum_{n \leq z / 10}\left(\frac{x}{n^{l}}\right)^{\alpha_{k}+\varepsilon}+x^{1 / l} \delta\left(\frac{z}{10}\right)(\log x)^{C_{k}} \\
& \ll_{l} \begin{cases}x^{\alpha_{k}+2 \varepsilon}+x^{1 / l} \delta(z / 10)(\log x)^{C_{k}} & \text { if } l \alpha_{k} \geq 1-l \varepsilon, \\
x^{\alpha_{k}+\varepsilon}(z / 10)^{1-l \alpha_{k}-l \varepsilon}+x^{1 / l} \delta(z / 10)(\log x)^{C_{k}} & \text { if } l \alpha_{k}<1-l \varepsilon\end{cases} \\
& \ll_{l, \varepsilon} \begin{cases}x^{\alpha_{k}+2 \varepsilon} & \text { if } l \alpha_{k} \geq 1-l \varepsilon, \\
x^{1 / l} & \text { if } l \alpha_{k}<1-l \varepsilon,\end{cases}
\end{aligned}
$$

since, for $x \geq e^{l e^{l}}$, we note that

$$
\begin{aligned}
x^{1 / l} \delta\left(\frac{z}{10}\right)(\log x)^{C_{k}} & =x^{1 / l} \delta\left(\frac{x^{1 / l}}{10}\right)(\log x)^{C_{k}} \\
& \ll x^{1 / l} \exp \left(-C(\log x)^{3 / 5}(\log \log x)^{-1 / 5}\right) .
\end{aligned}
$$

This proves Theorem 1 .

Proof of Theorem 2. We choose here $\varrho=\left(\delta\left(x^{1 / l}\right)\right)^{1 / 10}$ and note that $z=x^{1 / l}$. Set

$$
f(x):=\varrho z=x^{1 / l}\left(\left(\delta\left(x^{1 / l}\right)\right)^{1 / 10}\right) .
$$

From Lemma 3.3, we have

$$
\begin{aligned}
E_{k, l}(x) & \ll \sum_{n \leq \varrho x^{1 / l}}\left|\Delta_{k}\left(\frac{x}{n^{l}}\right)\right|+x^{1 / l} \varrho^{1-l} \delta\left(\varrho x^{1 / l}\right)\left(\log \left(\max \left(\frac{1}{\varrho}, x\right)\right)\right)^{C_{k}} \\
& \ll E_{1}+E_{2} .
\end{aligned}
$$

Without loss of generality the constant $A$ in (1.12) can be taken to be $<1$. Note that $x \geq e^{l e^{l}}$. Now, we observe that

$$
f(x):=\varrho z=x^{1 / l}\left(\left(\delta\left(x^{1 / l}\right)\right)^{1 / 10}\right) \geq x^{1 / 2 l}
$$

if $x \geq e^{l^{1 / 2}}$; but we have already assumed that $x \geq e^{l e^{l}}$. Since the function $\delta$ is decreasing, we find that

$$
\delta(\varrho z) \leq \delta\left(x^{1 / 2 l}\right)
$$

Note that

$$
\varrho^{1-l} \delta(\varrho z) \leq \varrho^{-1} \delta(\varrho z) \leq \delta\left(x^{1 / 2 l}\right)\left(\delta\left(x^{1 / l}\right)\right)^{-1 / 10}
$$




$$
\begin{aligned}
= & \exp \left(-A\left(\log \left(x^{1 / 2 l}\right)\right)^{3 / 5}\left(\log \log \left(x^{1 / 2 l}\right)\right)^{-1 / 5}\right) \\
& \times \exp \left(\frac{A}{10}\left(\log \left(x^{1 / l}\right)\right)^{3 / 5}\left(\log \log \left(x^{1 / l}\right)\right)^{-1 / 5}\right) \\
\leq & \exp \left(-C(\log x)^{3 / 5}(\log \log x)^{-1 / 5}\right),
\end{aligned}
$$

provided $x \geq e^{l^{2}}$. Hence, clearly,

$$
\frac{1}{Y} \int_{Y}^{2 Y} E_{2}^{2} d x \ll Y^{2 / l} \exp \left(-C(\log Y)^{3 / 5}(\log \log Y)^{-1 / 5}\right) \text {. }
$$

We note that for $Y \leq x \leq 2 Y$, we have $f(x) \leq f(2 Y)$. Now,

$$
\begin{aligned}
I_{1} & :=\int_{Y}^{2 Y} E_{1}^{2} d x=\int_{Y}^{2 Y}\left(\sum_{n \leq \varrho x^{1 / l}}\left|\Delta_{k}\left(\frac{x}{n^{l}}\right)\right|\right)^{2} d x \\
& \ll \int_{Y}^{2 Y}\left(\left|\Delta_{k}(x)\right|+\left|\Delta_{k}\left(\frac{x}{2^{l}}\right)\right|+\cdots+\left|\Delta_{k}\left(\frac{x}{[f(x)]^{l}}\right)\right|\right)^{2} d x \\
& +\int_{Y}^{2 Y}\left(\frac{x}{[f(x)]^{l}}\right)^{2\left(\alpha_{k}+\varepsilon\right)} d x \\
& \ll \int_{Y}^{2 Y}\left(\left|\Delta_{k}(x)\right|+\left|\Delta_{k}\left(\frac{x}{2^{l}}\right)\right|+\cdots+\left|\Delta_{k}\left(\frac{x}{[f(x)]^{l}}\right)\right|\right)^{2} d x+Y^{1+10 \varepsilon} .
\end{aligned}
$$

We note that (for $Y \geq 100 e^{l e^{l}}$ ),

$$
\left(\delta\left((2 Y)^{1 / l}\right)\right)^{(1 / 10)\left(1-l \beta_{k}-l \varepsilon / 2\right)} \leq \exp \left(-C(\log Y)^{3 / 5}(\log \log Y)^{-1 / 5}\right),
$$

provided $1-l \beta_{k}-l \varepsilon / 2>0$.

Therefore, from (4.6) and the Minkowski inequality (see item 200 of [6]), we get (using the inequality $(a+b)^{1 / 2} \leq a^{1 / 2}+b^{1 / 2}$ for $a \geq 0$ and $b \geq 0$ and the definition (1.11))

$$
\begin{aligned}
& I_{1}^{1 / 2} \ll \sum_{n \leq f(2 Y)}\left\{\int_{Y}^{2 Y}\left(\Delta_{k}\left(\frac{x}{n^{l}}\right)\right)^{2} d x\right\}^{1 / 2}+Y^{1 / 2+5 \varepsilon} \\
& \ll \sum_{n \leq f(2 Y)} n^{l / 2}\left(\frac{Y}{n^{l}}\right)^{(1 / 2)\left(1+2 \beta_{k}+\varepsilon\right)}+Y^{1 / 2+5 \varepsilon} \\
& \ll Y^{1 / 2+\beta_{k}+\varepsilon / 2 \sum_{n \leq f(2 Y)} n^{-l \beta_{k}-l \varepsilon / 2}+Y^{1 / 2+5 \varepsilon}} \\
& \ll \begin{cases}Y^{1 / 2+\beta_{k}+\varepsilon}+Y^{1 / 2+5 \varepsilon} & \text { if } l \beta_{k} \geq 1-l \varepsilon / 2, \\
Y^{1 / 2+\beta_{k}+\varepsilon / 2}(f(2 Y))^{1-l \beta_{k}-l \varepsilon / 2}+Y^{1 / 2+5 \varepsilon} & \text { if } l \beta_{k}<1-l \varepsilon / 2\end{cases} \\
& \ll \begin{cases}Y^{1 / 2+\beta_{k}+\varepsilon} & \text { if } l \beta_{k} \geq 1-l \varepsilon / 2, \\
Y^{1 / 2+1 / l} \exp \left(-C(\log Y)^{3 / 5}(\log \log Y)^{-1 / 5}\right) & \text { if } l \beta_{k}<1-l \varepsilon / 2 .\end{cases}
\end{aligned}
$$


Hence, we obtain

$$
\frac{I_{1}}{Y} \ll \begin{cases}Y^{2 \beta_{k}+2 \varepsilon} & \text { if } l \beta_{k} \geq 1-l \varepsilon / 2 \\ Y^{2 / l} \exp \left(-C(\log Y)^{3 / 5}(\log \log Y)^{-1 / 5}\right) & \text { if } l \beta_{k}<1-l \varepsilon / 2\end{cases}
$$

This proves Theorem 2.

REmark. From the work of Heath-Brown (see [7] and [8]), we know that $\beta_{4}=3 / 8$. If we fix $k=4$ and $l=2$ in Theorem 2 , then we find that $l \beta_{4}=3 / 4<1$, and hence the inequality (1.7) follows from Theorem 2 .

Acknowledgements. This project was carried out and completed when the second author visited the Department for Number Theory and Probability Theory, University of Ulm, Germany in 2004; he wishes to thank the University of Ulm for its warm hospitality and generous support. The authors are grateful to the referee for some useful comments.

\section{References}

[1] R. Balasubramanian and K. Ramachandra, Some problems of analytic number theory, II, Studia Sci. Math. Hungar. 14 (1979), 193-202.

[2] - - - Some problems of analytic number theory, III, Hardy-Ramanujan J. 4 (1981), $13-40$.

[3] R. Balasubramanian, K. Ramachandra and M. V. Subbarao, On the error function in the asymptotic formula for the counting function of $k$-full numbers, Acta Arith. 50 (1988), 107-118.

[4] K. Chandrasekharan, Introduction to Analytic Number Theory, Grundlehren Math. Wiss. 148, Springer, New York, 1968.

[5] K. Chandrasekharan and A. Good, On the number of integral ideals in Galois extensions, Monatsh. Math. 95 (1983), 99-109.

[6] G. H. Hardy, J. E. Littlewood and G. Pólya, Inequalities, 2nd ed., Cambridge Univ. Press, 1952.

[7] D. R. Heath-Brown, The twelfth power moment of the Riemann zeta-function, Quart. J. Math. Oxford Ser. (2) 29 (1978), 443-462.

[8] - Mean values of the zeta function and divisor problems, in: Recent Progress in Analytic Number Theory (Durham, 1979), Vol. I, Academic Press, London, 1981, $115-119$.

[9] M. N. Huxley, Exponential sums and lattice points, II, Proc. London Math. Soc. (3) 66 (1993), 279-301.

[10] A. Ivić, The Riemann Zeta-Function. The Theory of the Riemann Zeta-Function with Applications, Wiley, New York, 1985.

[11] M. Jutila, Riemann's zeta-function and the divisor problem, Ark. Mat. 21 (1983), 75-96.

[12] G. Kolesnik, On the estimation of multiple exponential sums, in: Recent Progress in Analytic Number Theory (Durham, 1979), Vol. I, Academic Press, London, 1981, $231-246$.

[13] —, On the method of exponent pairs, Acta Arith. 45 (1985), 115-143. 
[14] M. Kühleitner, On a question of A. Schinzel concerning the sum $\sum_{n \leq x}(r(n))^{2}$, Grazer Math. Ber. 318 (1993), 63-67.

[15] M. Kühleitner and W. G. Nowak, An omega theorem for a class of arithmetic functions, Math. Nachr. 165 (1994), 79-98.

[16] - - - The average number of solutions of the Diophantine equation $u^{2}+v^{2}=w^{3}$ and related arithmetic functions, Acta Math. Hungar. 104 (2004), 225-240.

[17] H. L. Montgomery and R. C. Vaughan, The distribution of squarefree numbers, in: Recent Progress in Analytic Number Theory (Durham, 1979), Vol. I, Academic Press, London, 1981, 247-256.

[18] K. Ramachandra, A remark on Perron's formula, J. Indian Math. Soc. 65 (1998), $145-151$.

[19] - , Notes on prime number theorem, I, in: Number Theory, R. P. Bambah, V. C. Dumir, R. J. Hans-Gill (eds.), Hindustan book Agency and Indian National Science Academy, 1999, 351-370.

[20] K. Ramachandra and A. Sankaranarayanan, On an asymptotic formula of Srinivasa Ramanujan, Acta Arith. 109 (2003), 349-357.

[21] S. Ramanujan, Some formulae in the analytic theory of numbers, Messenger of Math. 45 (1916), 81-84.

[22] —, Ramanujan's Papers, B. J. Venkatachala et al. (eds.), Prism Books, Bangalore, 2000, 169-173.

[23] A. Sankaranarayanan and K. Srinivas, On a method of Balasubramanian and Ramachandra (on the abelian group problem), Rend. Sem. Mat. Univ. Padova 97 (1997), 135-161.

[24] A. Schinzel, On an analytic problem considered by Sierpiński and Ramanujan, in: New Trends in Probability and Statistics (Palanga, 1991), Vol. 2, VSP, Utrecht, 1992, 165-171.

[25] D. Suryanarayana and R. Sitaramachandra rao, On an asymptotic formula of Ramanujan, Math. Scand. 32 (1973), 258-264.

[26] E. C. Titchmarsh, The Theory of the Riemann Zeta-Function, 2nd ed. (edited by D. R. Heath-Brown), Clarendon Press, Oxford, 1986.

[27] A. Walfisz, Weylsche Exponentialsummen in der neueren Zahlentheorie, Mathematische Forschungsberichte 15, Berlin, 1963.

[28] B. M. Wilson, Proofs of some formulae enunciated by Ramanujan, Proc. London Math. Soc. (2) 21 (1922), 235-255.

Department for Number Theory and Probability Theory

University of Ulm

D-89069 Ulm, Germany

E-mail: hamaier@mathematik.uni-ulm.de

School of Mathematics TIFR

Homi Bhabha Road Mumbai, 400 005, India E-mail: sank@math.tifr.res.in 